03

\title{
Электрооптический отклик ячеек с разной толщиной гомеопланарных слоев нематического жидкого кристалла при наклонном падении света на слой
}

\section{(C) А.А. Каретников, А.П. Ковшик, Н.А. Каретников, Е.И. Рюмцев, Е.В. Аксенова, А.В. Сванидзе}

Санкт-Петербургский государственный университет, 199034 Санкт-Петербург, Россия

e-mail: akaret@mail.ru

Поступила в редакцию 17.09.2020 г.

В окончательной редакции 17.09.2020 г.

Принята к публикации 28.10.2020 г.

Исследована электроуправляемая рефракция света в ячейках с различной толщиной слоя гомеопланарно ориентированного нематического жидкого кристалла (ЖК) для углов падения света на слой, превышающих угол полного внутреннего отражения обыкновенного луча $\delta_{0}$. Для ячеек с толщиной слоев жидкого кристалла $d=7,14$ и $22 \mu$ m получены зависимости времени нарастания и спада оптического отклика $\tau_{\text {on }}$ и $\tau_{\text {off }}$ от напряженности электрического поля и углов падения света $\delta$ на слой ЖК.

Ключевые слова: нематический жидкий кристалл, электрическое поле, рефракция.

DOI: $10.21883 / \mathrm{OS} .2021 .02 .50557 .234-20$

\section{Введение}

В недавно опубликованной работе [1] приведены результаты экспериментального исследования электрооптических свойств жидкокристаллической ячейки с гомеопланарной ориентацией директора и толщиной слоя $d=13.8 \mu \mathrm{m}$. При углах падения света на слой жидкого

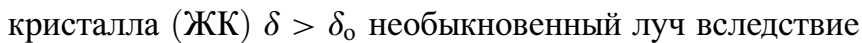
уменьшения его показателя преломления в направлении распространения испытывал поворот внутри слоя ЖК, подобный полному внутреннему отражению на границе двух сред. Воздействие управляющего электрического напряжения $U$ на слой ЖК искажало конфигурацию директора (нарушалось условие поворота), и свет проходил через ячейку. Были получены зависимости времен нарастания и спада оптического отклика $\tau_{\text {оn }}$ и $\tau_{\text {off }}$ от угла падения $\delta$ луча на слой ЖК. Это давало возможность изучать динамику электрооптического отклика для различной глубины проникновения $z^{*}=z^{*}(\delta)$ луча в слой. Для углов падения света $\delta$, значительно превышающих угол полного внутреннего отражения обыкновенного луча $\delta_{\mathrm{o}}$, времена $\tau_{\text {off }}$ составляли $1-2 \mathrm{~ms}$ и были на три порядка меньше времени релаксации оптического отклика в случае падения луча по нормали $\left(\tau_{\text {off }} \approx 1 \mathrm{~s}\right)$. Обнаруженные в указанной работе электрооптические свойства гомеопланарной ячейки могут найти применение в технологиях ЖК-дисплеев [2] и оптических переключателях для планарных волноводов [3]. В связи с этим целью настоящей работы является экспериментальное исследование электроуправляемой рефракции в ЖКячейках с гомеопланарной ориентацией при вариации толщины слоя ЖК, угла падения света и напряженности электрического поля $E=U / d$.

\section{Методика эксперимента}

Ячейки состояли из двух трапецеидальных стеклянных призм с показателем преломления $n_{g}=1.7125$ для $\lambda=632.8 \mathrm{~nm}$ (рис. 1 ).

Призмы имели основания размером $52 \times 24 \mathrm{~mm}$ и высоту $18 \mathrm{~mm}$. Для вариации в широких пределах угла падения луча на слой ЖК наклон боковых граней к основаниям составлял $68.0^{\circ}$. На поверхности оснований были нанесены прозрачные токопроводящие электроды. Один из электродов был покрыт гомеотропно ориентирующим слоем, полученным из раствора стеарилхлорида хрома в изопропиловом спирте методом центрифугирования при скорости вращения призмы в $3000 \mathrm{rpm}$. На поверхности другого электрода тем же методом наносилось полимерное покрытие. Планарная ориентация директора нематического ЖК на слое полимера создавалась с помощью натирания покрытия
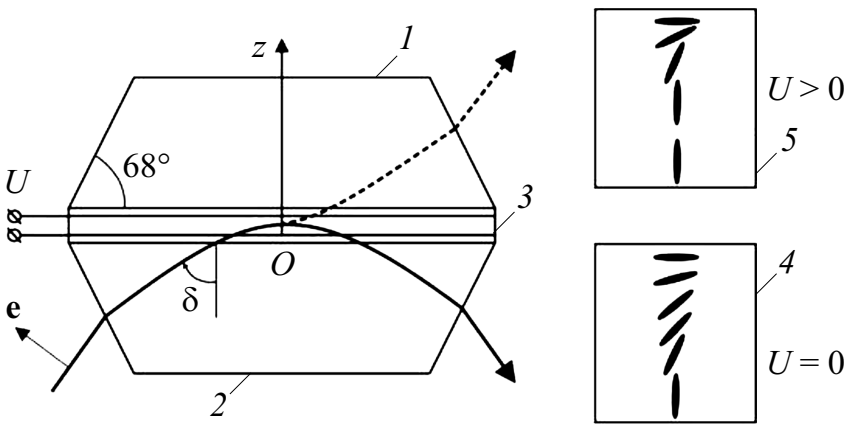

Рис. 1. ЖК-ячейка. $1,2-$ стеклянные призмы, 3 - слой ЖК, 4,5 - профиль директора при $U=0$ и $U>0$ соответственно, e - вектор поляризации лазерного луча, $z$ - нормаль к плоскости слоя ЖК. 
хлопковой тканью в одном направлении вдоль длинной оси основания призмы. Полимерное покрытие обеспечивало жесткое сцепление директора с поверхностью. Угол между директором и поверхностью слоя ЖК, определенный поляриметрическим методом [4], составлял не более $1^{\circ}$. Слои ЖК толщиной $d=7,14$ и $22 \mu \mathrm{m}$ размещались между основаниями призм (рис. 1). В работе использовалась нематическая смесь ЖК-1466 (НИОПИК) с показателями преломления $n_{o}=1.511$ для обыкновенного и $n_{\mathrm{e}}=1.691$ для необыкновенного лучей $(\lambda=632.8 \mathrm{~nm})$ при температуре $t=20^{\circ} \mathrm{C}$. Смесь имела положительную анизотропию диэлектрической проницаемости $\Delta \varepsilon=+11.7$ в интервале частот $1-100 \mathrm{kHz}$, и модули упругости $K_{11}=12 \mathrm{pN}$ и $K_{33}=0.99 K_{11}$.

В экспериментальной установке источником поляризованного излучения являлся гелий-неоновый лазер с длиной волны $632.8 \mathrm{~nm}$ и диаметром пучка $1 \mathrm{~mm}$. Требуемое направление вектора поляризации лазерного луча устанавливалось с помощью четвертьволновой пластинки и поляризатора, размещенного после нее. Далее луч направлялся на входную наклонную грань ЖК ячейки, расположенной на вращающемся столике с угловым отсчетным устройством.

На ячейки подавался управляющий одиночный импульс длительностью $200 \mathrm{~ms}$ с заполнением прямоугольным двуполярным напряжением с частотой $f=10 \mathrm{kHz}$ или последовательность управляющих импульсов с различной частотой следования от генератора Agilent 33522A через широкополосный усилитель Tabor Electronics 9100. Для регистрации интенсивности света, прошедшего через ячейку при воздействии электрического импульса, использовался фотоприемник с быстродействием $10^{-5} \mathrm{~s}$. Управляющий импульс и электрооптический отклик ячейки регистрировались с помощью цифрового двухканального осциллографа АСК3106. Время включения электрооптического отклика $\tau_{\text {оn }}$ определялось как интервал между моментом включения электрического импульса и выходом светопропускания на уровень 0.9 от установившегося уровня пропускания. Время выключения $\tau_{\text {off }}$ определялось как интервал между моментом выключения электрического импульса и снижением светопропускания от установившегося уровня до 0.1 от его значения.

\section{Распространение света в ЖК}

При исследовании рефракции луч лазера (рис. 1) направлялся на гомеотропно ориентированную поверхность слоя ЖК под углами $\delta>\delta_{\text {o }}=$ $=\arcsin \left(n_{o} / n_{g}\right)=61.9^{\circ}\left(\delta_{\text {o }}\right.$ - угол полного внутреннего отражения обыкновенного луча на границе раздела стекло-ЖК). Вектор поляризации света е и директор ЖК были расположены в плоскости падения, так что падающий на слой ЖК луч являлся необыкновенным (рис. 1). Луч входил в слой и испытывал полное внутреннее отражение внутри слоя на глубине

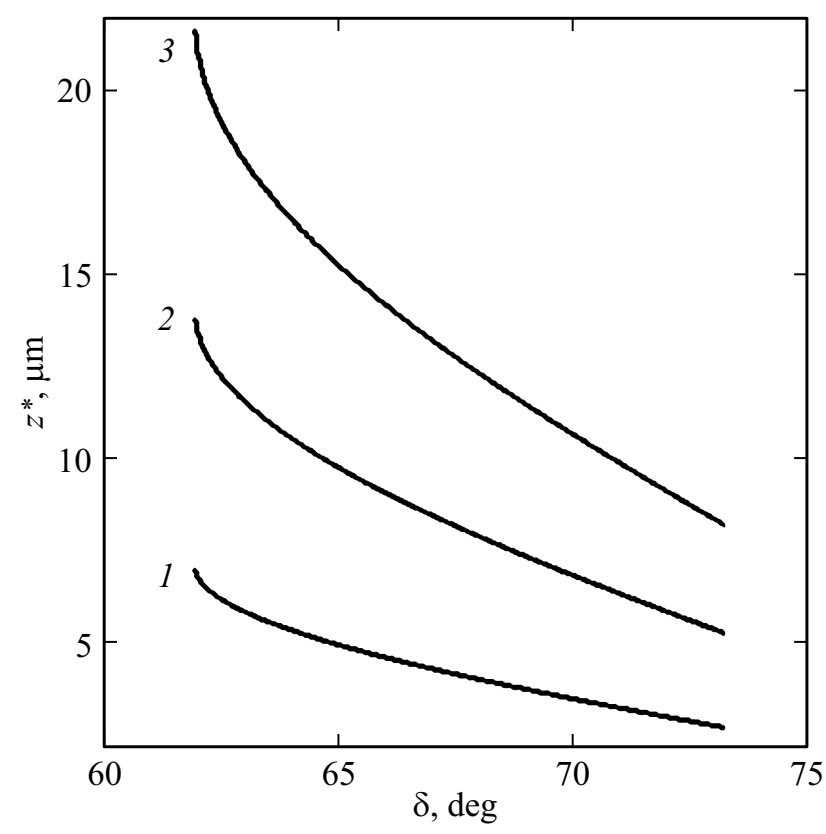

Рис. 2. Зависимости глубины проникновения необыкновенного луча $z^{*}$ от угла падения этого луча $\delta$ на гомеопланарный слой ЖК для $d=7$ (1), 14 (2), $22 \mu \mathrm{m}(3)$.

проникновения $z^{*}$, которую можно рассчитать, зная конфигурацию директора. Для определения конфигурации директора достаточно решить уравнения Эйлера-Лагранжа в одноконстантном приближении, так как используемый ЖК обладает близкими значениями упругих модулей Франка, характеризующих деформации поперечного и продольного изгибов $K_{33}=0.99 K_{11}$. В отсутствие внешнего электрического поля и с учетом жестких граничных условий угол $\theta$, который определяет отклонение директора от оси $O Z$, является линейной функцией $\theta=\pi z / 2 d$. Тогда величина $z^{*}$ для гомеопланарной ячейки связана с углом падения $\delta$ и показателями преломления ЖК $n_{e}$ и $n_{o}$ следующим соотношением:

$$
z^{*}=\frac{2 d}{\pi} \arccos \sqrt{\frac{n_{g}^{2} \sin ^{2} \delta-n_{o}^{2}}{n_{g}^{2}-n_{o}^{2}}} .
$$

В отличие от выражения для глубины проникновения, использованного в работе [1], здесь дополнительно учтено, что направления волнового и лучевого векторов не совпадают в точке полного внутреннего отражения. Однако параметры исследуемого ЖК таковы, что учет этого факта дает отличие в расчетах не более чем на $9 \%$.

С использованием формулы (1) были рассчитаны зависимости $z^{*}$ от $\delta$ в интервале углов падения 62.7-73.2 для ячеек с толщиной слоев ЖК $d=7,14$ и $22 \mu \mathrm{m}$. Полученные зависимости представлены на рис. 2.

Из рис. 2 видно, что увеличение угла падения $\delta$ необыкновенного луча на слой ЖК приводит к уменьшению глубины проникновения $z^{*}$ этого луча в слой. При этом относительная глубина проникновения $z_{R}=z^{*} / d$ 
не зависит от толщины слоя для одинаковых значений $\delta$ в соответствии с формулой (1).

\section{Результаты эксперимента}

В работе при подаче одиночного управляющего импульса на ЖК ячейки были получены зависимости времен нарастания $\tau_{\text {on }}$ и спада $\tau_{\text {off }}$ от напряженности электрического поля $E$ при угле падения $\delta=68.0^{\circ}$, соответствующем середине использованного интервала углов (рис. 3).

Анализ полученных экспериментальных данных пока-

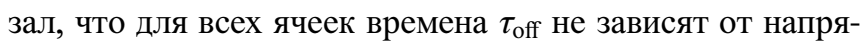
женности поля $E$ и находятся в интервале $1-2 \mathrm{~ms}$. При напряженностях поля $E>2.5 \mathrm{~V}_{\mathrm{pp}} / \mu \mathrm{m}$ времена включения $\tau_{\text {on }}$ снижаются от десятков миллисекунд до значений $1-2 \mathrm{~ms}$, близких к значениям $\tau_{\text {off }}$.

Исследование зависимости $\tau_{\text {on }}$ и $\tau_{\text {off }}$ от глубины проникновения $z^{*}$ луча в слой ЖК проводилось при максимальной напряженности электрического поля $E=2.9 \mathrm{~V}_{\mathrm{pp}} / \mu \mathrm{m}$, соответствующей минимальным значениям времен $\tau_{\text {on }}$ и $\tau_{\text {off. }}$ Угол падения луча света на слой ЖК в ячейках варьировался в интервале от 62.7 до $73.2^{\circ}$. При этом величина $z^{*}$ изменялась от 5.8 до $2.4 \mu \mathrm{m}$ для ячейки с толщиной $d=7 \mu \mathrm{m}$, от 11.4 до $4.6 \mu \mathrm{m}$ для $d=14 \mu \mathrm{m}$ и от 18.3 до $7.4 \mu \mathrm{m}$ для $d=22 \mu \mathrm{m}$. Для исследованных ячеек зависимости $\tau_{\text {on }}$ и $\tau_{\text {off }}$ от глубины проникновения луча в слой $z^{*}$ при напряженности поля $E=2.9 \mathrm{~V}_{\mathrm{pp}} / \mu \mathrm{m}$ представлены на рис. 4 .

Из рисунка видно, что для всех ячеек значения $\tau_{\text {оп }}$ слабо зависят от глубины проникновения луча в слой ЖК, а

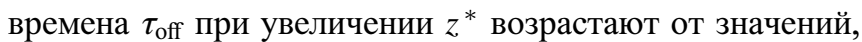
близких к $\tau_{\text {оn }}$, до нескольких десятков миллисекунд. Для детального анализа полученных результатов величины

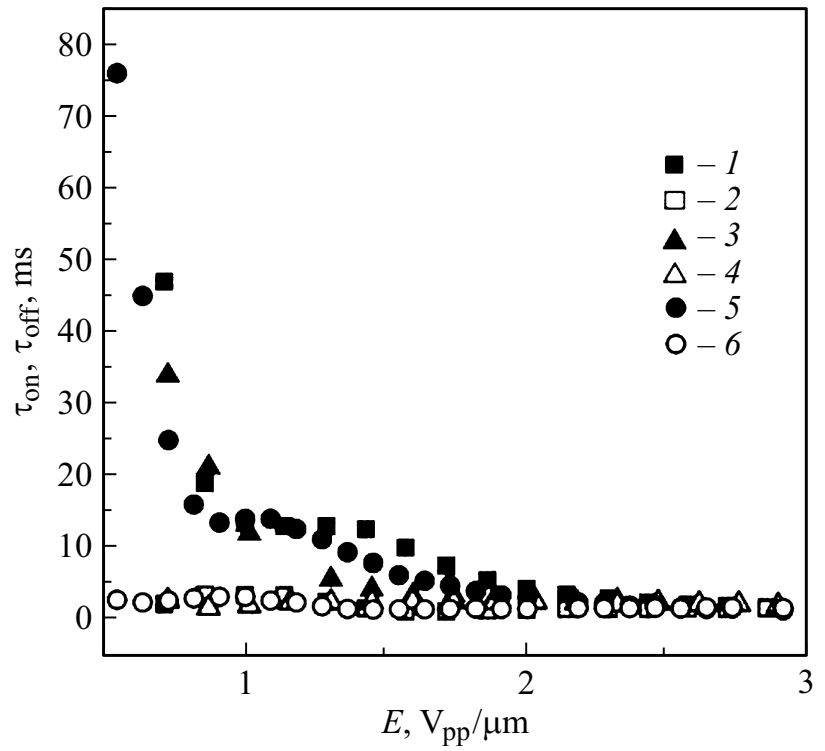

Рис. 3. Зависимости $\tau_{\text {on }}(1,3,5)$ и $\tau_{\text {off }}(2,4,6)$ оптических откликов ЖК ячеек от напряженности электрического поля $E$ для $\delta=68.0^{\circ} . d=7(1,2), 14(3,4), 22 \mu \mathrm{m}(5,6)$.

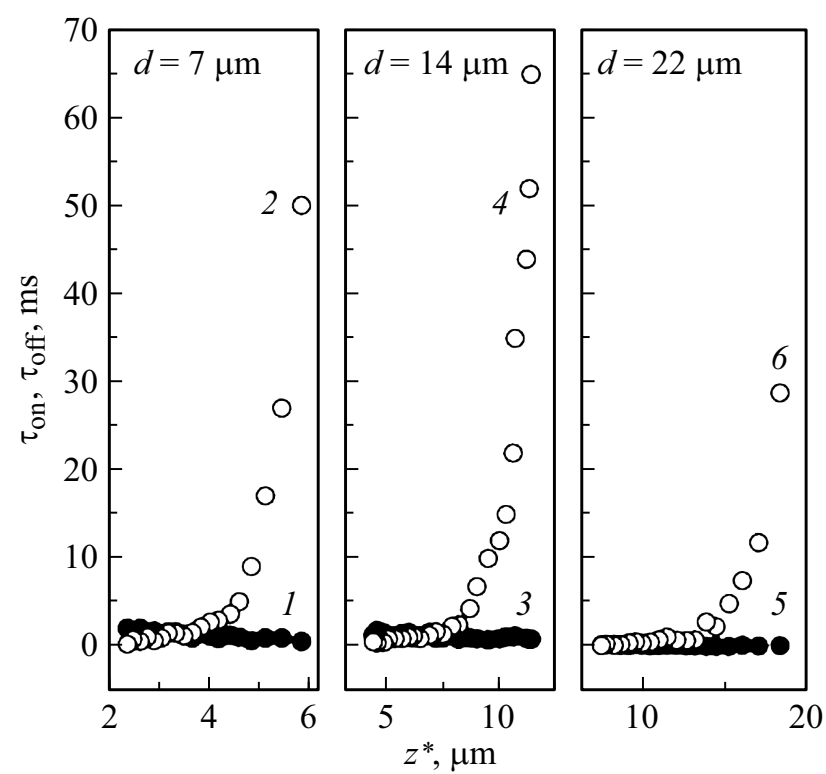

Рис. 4. Зависимость времен включения $\tau_{\text {оп }}(1,3,5)$ и выключения $\tau_{\text {off }}(2,4,6)$ электрооптического отклика от глубины проникновения луча $z^{*}$ в слои ЖК толщиной $d=7,14$ и $22 \mu \mathrm{m}$.

Угловые зависимости времен $\tau_{\text {on }}$ и $\tau_{\text {off }}$ ЖК-ячеек

\begin{tabular}{c|c|c|c|c|c|c|c}
\hline \multirow{2}{*}{$\delta$, deg } & \multirow{2}{*}{$z_{R}$} & \multicolumn{2}{|c|}{$d=7 \mu \mathrm{m}$} & \multicolumn{2}{c|}{$d=14 \mu \mathrm{m}$} & \multicolumn{2}{c}{$d=22 \mu \mathrm{m}$} \\
\cline { 3 - 7 } & & $\tau_{\text {on }}$ & $\tau_{\text {off }}$ & $\tau_{\text {on }}$ & $\tau_{\text {off }}$ & $\tau_{\text {on }}$ & $\tau_{\text {off }}$ \\
\hline 62.7 & 0.83 & 0.5 & 50 & 0.8 & 65 & 0.3 & 29 \\
63.9 & 0.73 & 0.9 & 17 & 0.9 & 12 & 0.4 & 7.7 \\
65.1 & 0.66 & 1.0 & 5.0 & 0.9 & 6.8 & 0.3 & 2.5 \\
66.3 & 0.59 & 0.8 & 2.9 & 0.8 & 2.5 & 0.3 & 1.0 \\
67.4 & 0.55 & 1.4 & 2.1 & 1.0 & 1.4 & 0.4 & 0.9 \\
68.6 & 0.50 & 1.3 & 1.1 & 1.6 & 1.3 & 0.3 & 1.0 \\
69.8 & 0.45 & 1.6 & 1.4 & 1.0 & 1.2 & 0.4 & 0.6 \\
70.9 & 0.41 & 1.7 & 0.6 & 1.5 & 0.9 & 0.4 & 0.7 \\
72.1 & 0.37 & 2.0 & 0.5 & 1.0 & 0.8 & 0.5 & 0.4 \\
73.2 & 0.34 & 2.0 & 0.2 & 1.8 & 0.4 & 0.4 & 0.3
\end{tabular}

$\tau_{\text {on }}$ и $\tau_{\text {off }}$ приведены в таблице при различных значениях угла $\delta$ и относительной глубины проникновения $z_{R}$.

Приведенные в таблице результаты показывают, что для ячеек с толщиной $d=7$ и $14 \mu \mathrm{m}$ при всех значениях $z_{R}$ величины $\tau_{\text {оn }}$ не превышают $2 \mathrm{~ms}$. В то же время $\tau_{\text {off }}$ для указанных ячеек уменьшаются и становятся меньше времен $\tau_{\text {on }}$ при уменьшении $z_{R}$. Для ячейки с наибольшей толщиной слоя ЖК $(d=22 \mu \mathrm{m})$ времена $\tau_{\text {on }}$ и $\tau_{\text {off }}$ оказались меньшими, чем $\tau_{\text {on }}$ и $\tau_{\text {off }}$ у двух других ячеек. В частности, время $\tau_{\text {оn }}$ находится в интервале $0.3-0.5 \mathrm{~ms}$ для всех относительных глубин

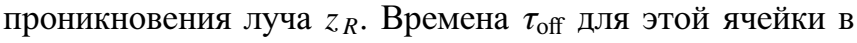
интервале $z_{R}$ от 0.83 до 0.59 уменьшаются от 29 до $1 \mathrm{~ms}$, а в интервале от 0.59 до 0.34 времена $\tau_{\text {off }}$ снижаются от 1.0 до $0.3 \mathrm{~ms}$. То обстоятельство, что значения $\tau_{\text {оn }}$ и

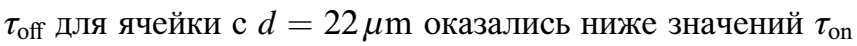
и $\tau_{\text {off }}$ для ячеек с $d=7$ и $14 \mu \mathrm{m}$, может быть связано 


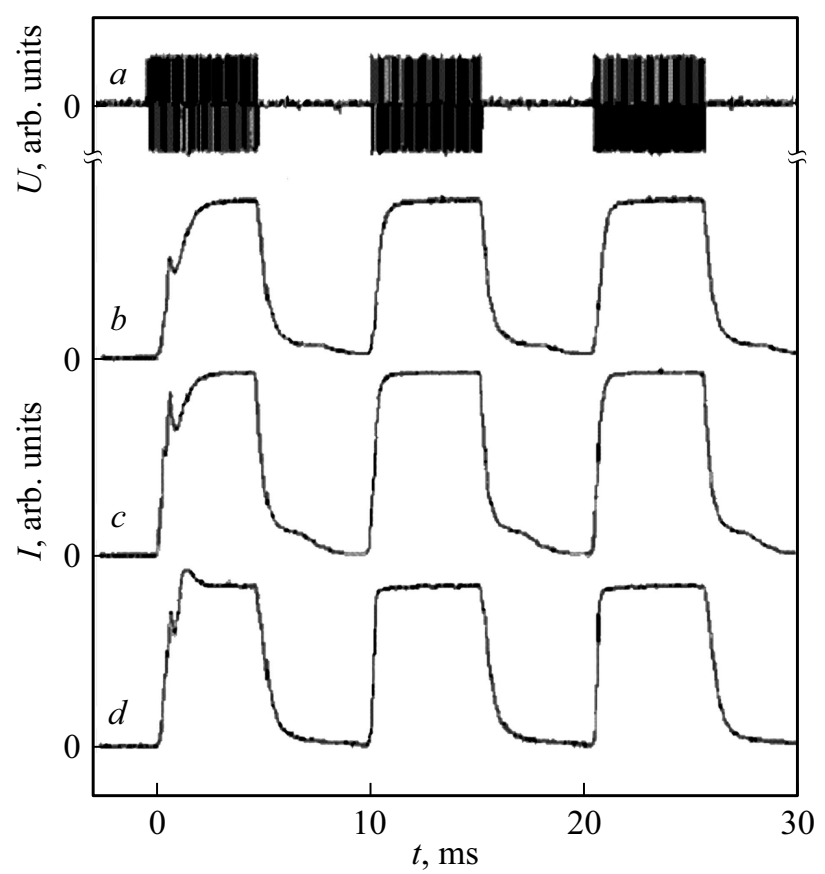

Рис. 5. (a) Управляющие электрические импульсы для $F=100 \mathrm{~Hz}, f=10 \mathrm{kHz}, E=2.9 \mathrm{~V}_{\mathrm{pp}} / \mu \mathrm{m}$. Оптические отклики ЖК ячеек: $d=7(b), 14(c), 22 \mu \mathrm{m}(d)$ при угле падения луча на слой $\delta=68.0^{\circ}$.

с ослаблением влияния обратных потоков в ЖК на оптический отклик в ячейке с большей толщиной слоя.

Приведенные результаты получены при воздействии на ЖК ячейку одиночного управляющего импульса. В то же время для технических применений ЖК может быть важна такая характеристика, как максимальная частота следования управляющих импульсов $F=F_{\max }$, до которой глубина амплитудной модуляции оптического отклика $m$ сохраняет значение $100 \%$. Глубина модуляции $m$ определяется соотношением

$$
m=\left(1-I_{\min } / I_{\max }\right) 100 \%,
$$

где $I_{\max }$ и $I_{\min }$ - максимальная и минимальная интенсивности прошедшего через ячейку света. Для определения $F_{\max }$ в нашем случае были исследованы осциллограммы оптических откликов ЖК ячеек при подаче управляющих импульсов с различной частотой следования (при частоте заполнения $f=10 \mathrm{kHz}$ и напряженности поля $\left.E=2.9 \mathrm{~V}_{\mathrm{pp}} / \mu \mathrm{m}\right)$. Осциллограммы были получены для угла падения света $\delta=68.0^{\circ}$ (середина углового диапазона) и максимального значения угла падения $\delta=73.2^{\circ}$. Было установлено, что для $\delta=68.0^{\circ}$ величина $F_{\max } \approx 100 \mathrm{~Hz}$ (рис. 5) и $F_{\max } \approx 300 \mathrm{~Hz}$ для $\delta=73.2^{\circ}$ (рис. 6). Большее значение $F_{\max }$ для угла падения света $\delta=73.2^{\circ}$ связано с тем, что для этого угла времена $\tau_{\text {off }}$

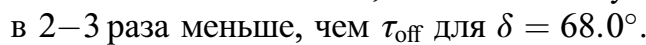

Наблюдающиеся искажения формы оптических откликов на рис. 5, 6, по-видимому, вызваны обратными потоками в ЖК. Искажения минимальны на откликах, начиная со второго (рис. 5) и третьего (рис. 6). Это объясняется тем, что каждый последующий электрический импульс накладывается на сформированную предыдущими импульсами (почти полностью гомеотропную) конфигурацию директора ЖК, которая не успевает вернуться к исходной гомеопланарной при прекращении воздействия электрического импульса.

Для сравнения динамических характеристик исследованной электроуправляемой рефракции и электроуправляемого двулучепреломления в тех же ячейках были измерены времена $\tau_{\text {off }}$ для нормального падения луча света на слой ЖК $\left(\delta=0^{\circ}\right)$ при той же напряженности электрического поля $E=2.9 \mathrm{~V}_{\mathrm{pp}} / \mu \mathrm{m}$. Ячейки размещались между скрещенными поляризаторами. Направление вектора поляризации падающего света составляло $45^{\circ} \mathrm{c}$ направлением директора на планарно ориентированной поверхности слоя ЖК. Для ячеек с толщиной слоев 7,14 и $22 \mu \mathrm{m}$ были получены значения $\tau_{\text {off }} \approx 0.2,1.0$ и $2.7 \mathrm{~s}$ соответственно. Эти значения $\tau_{\text {off }}$ на несколько по-

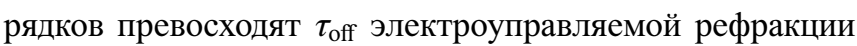
для углов падения $\delta$ в диапазоне $62.7-73.2^{\circ}$. Большие

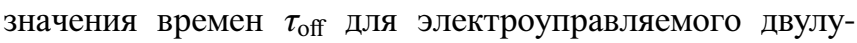
чепреломления обусловлены полной свободной переориентацией директора в исходное состояние во всем слое ЖК после выключения поля. Малые значения $\tau_{\text {off }}$ электроуправляемой рефракции, по-видимому, связаны с быстрым восстановлением ориентации директора в тонких пристеночных слоях у границы с планарной ориентацией ЖК. В работе [5] рекордная динамика электрооптического отклика в ячейке со скрещенными встречно-

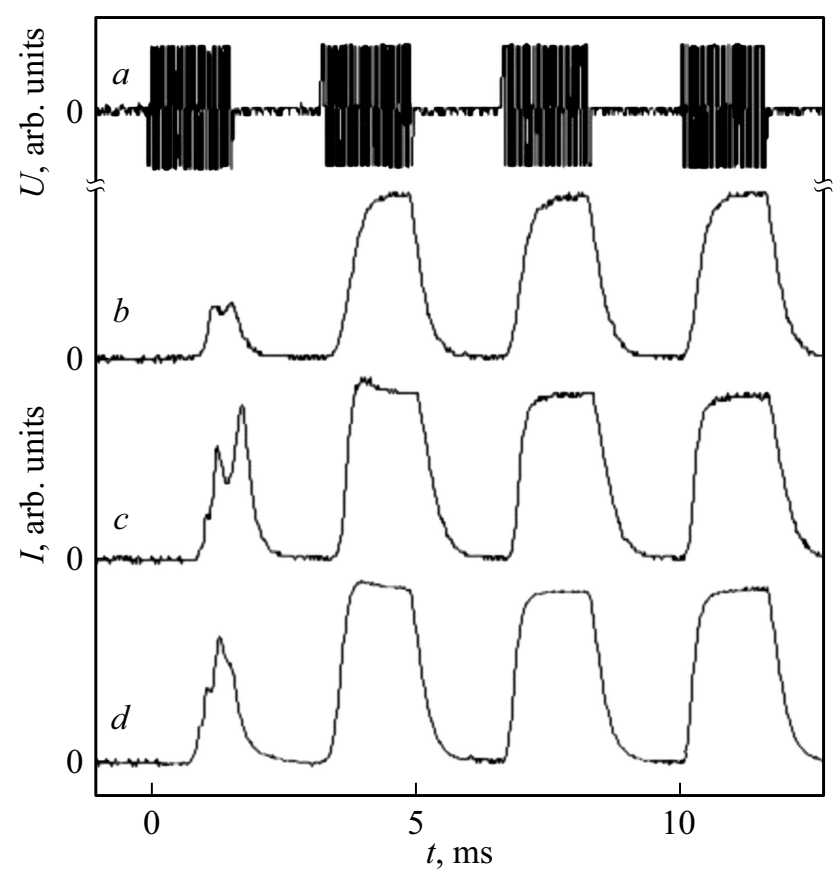

Рис. 6. (a) Управляющие электрические импульсы для $F=300 \mathrm{~Hz}, f=10 \mathrm{kHz}, E=2.9 \mathrm{~V}_{\mathrm{pp}} / \mu \mathrm{m}$. Оптические отклики ЖК ячеек: $d=7(b), 14(c), 22 \mu \mathrm{m}(d)$ при угле падения луча на слой $\delta=73.2^{\circ}$. 
штыревыми электродами также объясняется быстрым изменением ориентации директора в приповерхностном слое ЖК при переключении электрического поля.

\section{Заключение}

Проведенное исследование электроуправляемой рефракции в гомеопланарных слоях ЖК различной толщины $(d=7,14,22 \mu \mathrm{m})$ показало, что наименьшими временами нарастания и спада электрооптического отклика $\tau_{\text {on }}$ и $\tau_{\text {off }}$ обладает ячейка с максимальной толщиной слоя ЖК $d=22 \mu \mathrm{m}$. Это может быть связано с ослаблением влияния обратных потоков ЖК на оптический отклик в ячейке с большей толщиной слоя. Также было показано, что максимальная частота следования управляющих импульсов $F_{\max }$, до которой глубина амплитудной модуляции оптического отклика $m$ сохраняет значение $100 \%$, увеличивается от 100 до $300 \mathrm{~Hz}$ при увеличении угла падения $\delta$ от 62.7 до $73.2^{\circ}$ для исследованных ЖК ячеек.

Использование ЖК материалов с лучшими вязкоупругими характеристиками, очевидно, позволит повысить быстродействие устройств на эффекте электроуправляемой рефракции в гомеопланарных слоях ЖК.

\section{Конфликт интересов}

Авторы заявляют, что у них нет конфликта интересов.

\section{Список литературы}

[1] Каретников Н.А., Ковшик А.П., Каретников А.А., Рюмцев Е.И., Аксенова Е.В., Сванидзе А.В. // Письма в ЖЭТФ. 2017. Т. 106. В. 5. C. 293; Karetnikov N.A., Kovshik A.P., Karetnikov A.A., Ryumtsev E.I., Aksenova E.V., Svanidze A.V. // JETP Lett. 2017. V. 106. N 5. P. 313.

[2] Хи P., Ho J., Chigrinov V., Kwok H.S. // SID’08 Digest. 2008. V. 34. P. 1891.

[3] Zhang A., Chan K.T., Demokan M.S., Chan V.W.C., Chan P.C.H., Kwok H.S., Chan A.H.P. // Appl. Phys. Lett. 2005. V. 86. P. 211108.

[4] Karetnikov A.A., Karetnikov N.A., Kovshik A.P., Rjumtsev Y.I. New Method of Determination of the Tilt Angle at a Liquid Crystal-Glass Interface. [Электронный pecypc] Режим доступа: https://arxiv.org/abs/0910.2162v1

[5] Гейвандов А.Р., Симдянкин И.В., Барник М.И., Палто В.С., Палто С.П. // Крист. 2019. Т. 64. № 3. С. 407; Geivandov A.R., Simdyankin I.V., Barnik M.I., Palto V.S., Palto S.P. // Cryst. Report. 2019. V. 64. N 3. P. 433. 